\title{
Characterization of Fiber Bridging in Mode II Fracture Growth of Laminated Composite Materials
}

\author{
K.N. Anyfantis ${ }^{1, a}$, N.G. Tsouvalis ${ }^{1, b}$ \\ ${ }^{1}$ Shipbuilding Technology Laboratory, School of Naval Architecture and Marine Engineering, \\ National Technical University of Athens, Athens, Greece

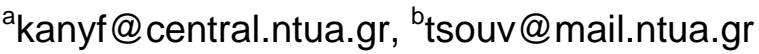

Keywords: Delamination growth, Bridging law, Laminated composites, Interface element

\begin{abstract}
The present study involves an experimental and analytical investigation of the Mode II delamination propagation and the fibre bridging effects incorporated in the Fracture Process Zone (FPZ). End Notch Flexure (ENF) specimens from a unidirectional glass/epoxy composite material have been fabricated and tested. In order to construct the fracture resistance curve (R-curve) of the ENF tests, three different data reduction schemes have been utilized. The fibre bridging effects in the FPZ have been addressed with the use of traction-separation laws, as extracted from the corresponding calculated R-curves. These laws can be used to describe the constitutive relationship in interface finite elements, for the numerical modelling of delamination growth in laminated composite structures.
\end{abstract}

\section{Introduction}

Fibre Reinforced Polymer (FRP) laminated composite materials have attracted a wide range of uses in civil, marine, automotive and aerospace applications on account of their superior properties. Nevertheless, due to their low interlaminar strength, they are susceptible to delamination damage, hence, extensive efforts have been devoted to the development of reliable test methods that can accurately measure delamination resistance of FRP materials.

Delamination resistance, which is usually expressed by the term "interlaminar fracture toughness", is often characterized in terms of the critical strain energy release rate, $G_{c}$. Interlaminar fracture toughness for delamination in opening Mode I is known as $G_{I c}$ and for the sliding shear Mode II as $G_{I I c}$. In recent years the DCB-UBM test (Double Cantilever Beam - DCB specimen loaded by uneven bending moments) has been used often for the characterization of mixed mode fracture of composite structures [1,2]. For the determination of the Mode I interlaminar fracture toughness of composite materials, the DCB test has been accepted as a standard method (ASTM D5528, ISO/DIS 15024). On the contrary, a commonly accepted standard for the measurement of $G_{I I c}$ has not yet been established. Despite this lack, several test methods are available for quantifying $G_{I I c}$ and have been widely used for research purposes. Among them, the End-NotchedFlexure (ENF), the End-Loaded-Split (ELS) and the Four-Point ENF (4ENF) tests are the most popular procedures found in the literature. The ENF test [3] is most widely used due to its simple fixture based on a conventional three point bending set up and has been adopted by the Japan Industrial Standards Group (JIS) as a standard test. On the other hand the ELS [4] and 4ENF [5] tests require a more complicated test set up and present some problems related to large friction effects [6]. Therefore, in the present study focus is given on the ENF test configuration.

Mode II fracture characterization tests involve difficulties regarding monitoring of the crack tip position, as the crack tends to close with the increase of the applied load [7]. The formation of a large fracture process zone (FPZ) behind the crack tip as crack propagates is characterized by the fibre bridging effect [2], which hinders the clear visualization of the crack tip.

From a structural point of view fibre bridging is beneficial, since it results in an increasing fracture resistance as crack grows. This increase in the fracture energy as crack propagates is described by a fracture resistance curve, namely the R-curve [8]. Resistance to delamination should not be based on the R-curve when a large FPZ is present. R-curves cannot be considered as a 
material property, since they depend on the geometry of the specimen. Instead, fibre bridging can be expressed in the form of a traction-separation law (bridging law), which can be inferred from the experimentally obtained R-curve [2]. In the case of Mode II fracture, a bridging law expresses the dependency between the closure traction (bridging stress) and the local crack sliding. When fracture toughness reaches a plateau in the R-curve at a specific value of crack sliding (steady-state interlaminar fracture toughness, $G_{s s}$ ), closure traction vanishes, leaving the crack faces free of traction [8].

The purpose of this work is to propose a characterization method of the FPZ in Mode II fracture of unidirectional glass/epoxy composite materials using bridging laws. The experimental programme involves fabrication and testing of ENF specimens, whereas post-processing of the experimental results is based on the construction of R-curves by applying three different data reduction schemes. The R-curves were used explicitly for the definition of the bridging laws.

\section{Experimental procedure}

Six layers of $600 \mathrm{gr} / \mathrm{m}^{2}$ unidirectional glass fabric were used together with epoxy resin D.E.R. 353 to manufacture a composite laminated plate. During manufacturing a PTFE film with $58 \mu \mathrm{m}$ thickness was inserted in the mid-plane for the creation of the delamination. The plate was manufactured by the hand lay up method and was cured at $25^{\circ} \mathrm{C}$ for 48 hours. After curing, five ENF specimens were cut out from the plate with waterjet. The composite system had a fibre weight fraction of 50\%, whereas its main Young's modulus $E_{l}$ was measured from standard tests and found to be $E_{1}=18000 \mathrm{MPa}$. Young's modulus $E_{3}$ and shear modulus $G_{13}$ were taken from the literature equal to 2200 and $1800 \mathrm{MPa}$, respectively.

The geometry and dimensions of the $140 \mathrm{~mm}$ long ENF specimens are given in Fig. 1. The thickness of the specimens is $2 h=4.55 \pm 0.1 \mathrm{~mm}$, their width is equal to $20 \mathrm{~mm}$ and their unsupported length $100 \mathrm{~mm}$. In order to achieve crack growth stability, the initial crack length $\alpha_{o}$ was chosen to be equal to $70 \%$ of the half unsupported length of the specimen [9], thus resulting in a value of $35 \mathrm{~mm}$.

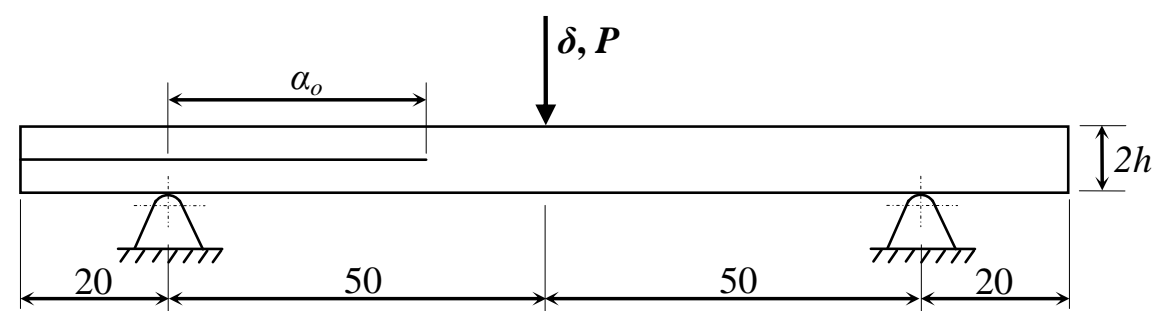

Fig. 1. Schematic view of the ENF test (dimensions in $\mathrm{mm}$ )

In an effort to monitor the crack length, thin vertical lines have been marked on the lateral sides of the specimens with a spacing of $2 \mathrm{~mm}$. Prior to the creation of these marks, a white correction fluid was applied for improvement of the crack tip visualization. Crack propagation was monitored by visual inspection of the crack length through the use of a high resolution (1920x1080) 60 frames per second video camera equipped with 16x optical zoom lens, recording the marked side of the specimens. ENF tests were displacement controlled with a speed of $0.5 \mathrm{~mm} / \mathrm{min}$ and were carried out in a $15 \mathrm{kN}$ capacity hydraulic testing machine. The load-deflection curve $(P-\delta)$ was recorded during each test as a function of time.

\section{Data reduction schemes}

Post processing of the experimental results obtained from an ENF test is based on the calculation of the critical fracture energy, $G_{I I c}$ and the corresponding R-curve. Most of the methods used for these calculations require the load-deflection $(P-\delta)$ data, together with the corresponding values of the crack length $\alpha$. In order to map the crack length on the $P$ - $\delta$ curve obtained from each test, the video 
was processed, measuring first the crack length as a function of time and then combining the registered time functions of $P$ and $\delta$ data with that of the crack length.

For the purpose of this study, three different data reduction schemes have been utilized for calculating the R-curves of the ENF specimens.

Compliance Calibration Method (CCM). CCM is a classical data reduction scheme for calculating $G_{I I c}[10]$ using the Irwin - Kies equation. According to this method, apart from loading and geometry, $G_{I I C}$ is also given as a function of specimen's compliance $C$, defined as deflection per unit load $(C=\delta / P)$. Thus, if a plot of $C$ versus $\alpha$ is drawn for any specimen geometry, its slope at a particular point $(C, \alpha)$ can be measured. In order to avoid any errors occurring from fitting a continuous function to the discrete points $(C, \alpha)$, a different approach is followed in the present study for the calculation of $d C / d \alpha$. By defining crack length $\alpha_{i}=\alpha_{o}+i \Delta \alpha$, where $\Delta \alpha$ is the crack increment and $i=0, \ldots, n$ ( 0 represents crack initiation and $n$ represents maximum number of crack tip measurements), the following formulas are derived:

$$
\left.\frac{d C}{d \alpha}\right|_{a=a_{0}, a_{n}}=\frac{C_{i}-C_{i-1}}{\alpha_{i}-\alpha_{i-1}}, \quad i=1 \text { or } n \quad,\left.\quad \frac{d C}{d \alpha}\right|_{a=a_{i}}=\frac{1}{2}\left(\frac{C_{i}-C_{i-1}}{\alpha_{i}-\alpha_{i-1}}+\frac{C_{i+1}-C_{i}}{\alpha_{i+1}-\alpha_{i}}\right), i=1, \ldots,(n-1)
$$

Corrected Beam Theory (CBT). This is another classical method proposed in [11] and based on a beam elastic foundation model that takes into account corrections of the measured crack length. According to this method, $G_{I I c}$ is given as a function of loading, geometry, crack length and material properties $E_{1}, E_{3}$ and $G_{13}[11]$.

Compliance Based Beam Model (CBBM). The above CCM and CBT data reduction schemes require accurate crack length measurements, which present difficulties in obtaining. Thus, a novel calculation method has been proposed [12], which is based on the beam theory and according to which the results depend only on the specimen's compliance during the tests and take into account several effects that affect the behaviour of the specimen and consequently the $P-\delta$ curve, which are neglected by the classical methods. $G_{I I c}$ is calculated by a modified Irwin - Kies equation as a function of loading, geometry and equivalent crack length that takes into account the FPZ influence.

\section{Experimental Results}

A deformed ENF specimen during the three-points bending test is shown in Fig. 2 and a magnified view of the crack tip area is shown in Fig. 3. A typical $P-\delta$ - $\alpha$ curve is presented in Fig. 4 . The stars on the graph indicate the crack length in $\mathrm{mm}$, as measured from the high resolution video camera by image processing techniques. It should be pointed out that crack initiated directly from the insert film and always propagated along the mid-plane of the specimens. As shown in Fig. 4, the experimental registered curve exhibits an initial linear response, followed by increasing nonlinearities as the crack begins to propagate. After the maximum load is attained, the slope of the load-deflection curve begins to soften together with delamination progression. The crack propagated in a stable manner during the displacement control fracture testing for all five specimens tested.

According to CCM, the compliance was calculated from $C_{i}=\delta_{i} / P_{i}$ for each value of $\alpha_{i}$. For the calculation of the slopes $(\mathrm{d} C / \mathrm{d} \alpha)$, Eq. 1 has been utilized. The average fracture toughness values calculated with the CCM approach are listed in Table 1. The same table presents also results from the calculation of the average fracture toughness with the use of the CBT and CBBM data reduction schemes. Fig. 5 presents the corresponding R-curves obtained from the average values of Table 1.

It is noteworthy that fracture toughness values obtained with CBT and CBBM schemes compare very well between each other, exhibiting also very good repeatability, since the coefficient of variance $(\mathrm{COV})$ for each crack length is rather low. On the other hand, $G_{I I c}$ values calculated with CCM are generally higher than those of the other two schemes, having also higher COVs. This behaviour is attributed to the fact that CCM does not take under consideration any crack corrections of the measured crack length $\alpha_{i}$ for the calculation of $G_{I I c}$, which in contrary CBT and CBBM 


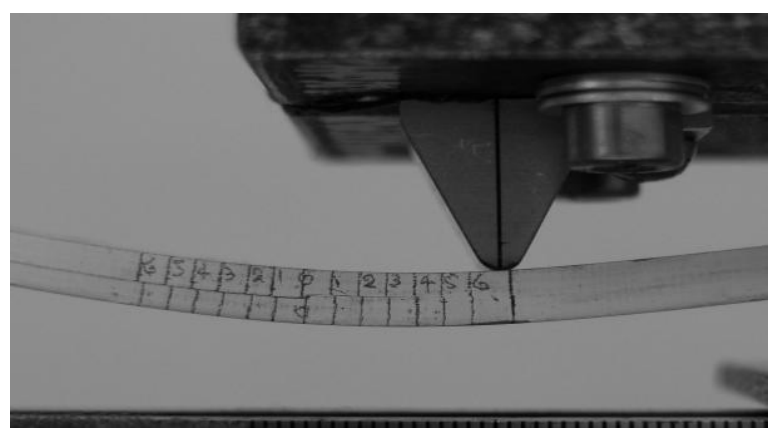

Fig. 2. Experimental setup

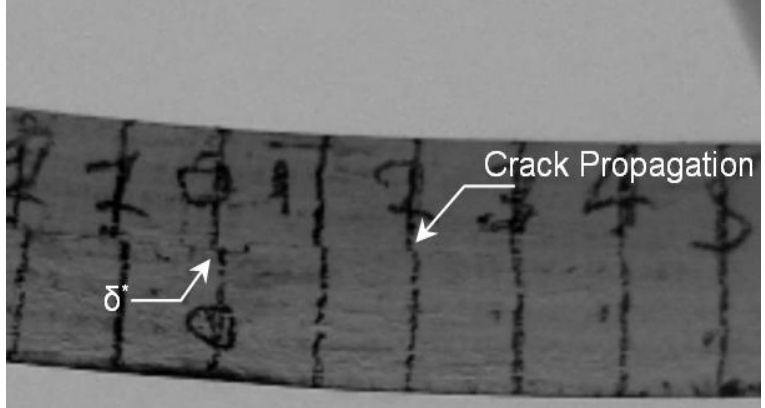

Fig. 3. Crack length measurement

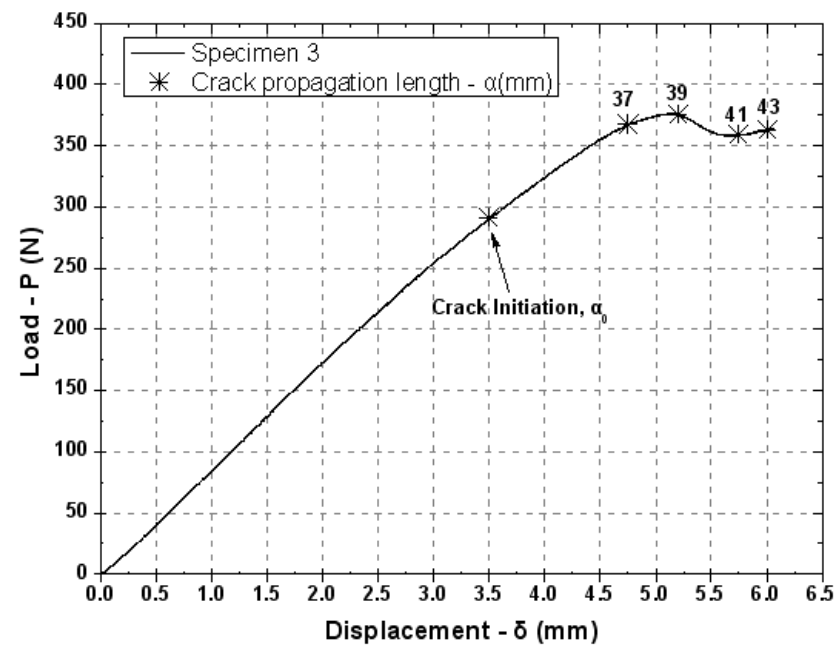

Fig. 4. Typical $P-\delta-\alpha$ curve of an ENF test

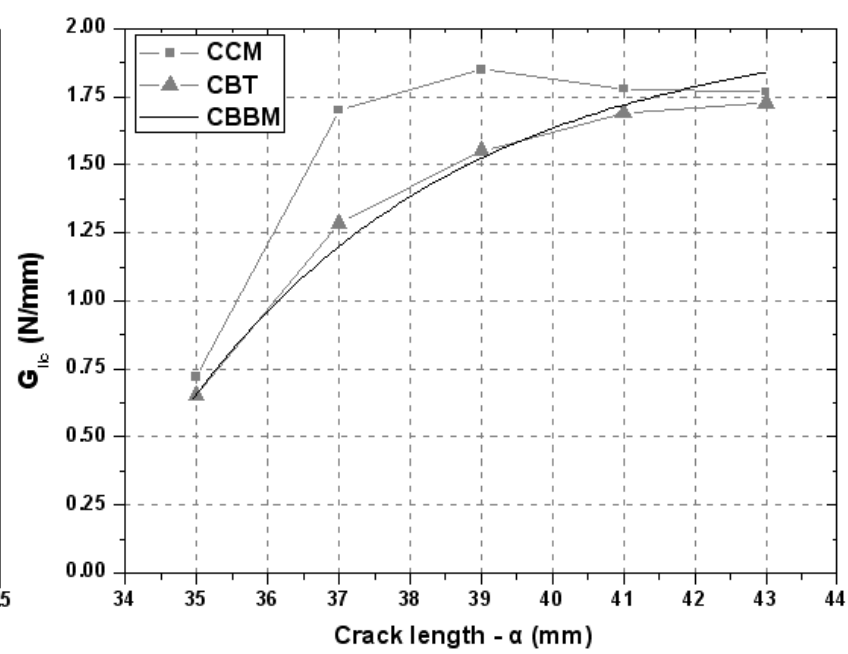

Fig. 5. Experimentally obtained R-curve

undertake. The R-curve obtained from CBBM has a continuous form, whereas the respective ones obtained from CCM and CBT have a discrete form. As Fig. 6 shows and Table 1 indicates, fracture toughness increases as the crack propagates, followed by a plateau region where it attains a steady state value, $G_{s s}$, for any further increase of the crack length. The same behaviour of the R-curves has been also reported from similar studies found in the literature [13]. In the R-curve obtained by CCM, steady state appears soon after crack initiation, whereas the transition is much smoother in the other two cases. In spite of the differences of the $G_{I I c}$ values between the CCM and the other two schemes, fracture toughness at crack initiation and at the steady state indicate very good repeatability among all three schemes, with a COV being approximately equal to $6 \%$.

\section{Characterization of the FPZ}

The extraction of a bridging law from the experimentally obtained R-curves can be done using formula

$$
\tau\left(\delta^{*}\right)=\frac{\partial G_{I I}}{\partial \delta^{*}}
$$

connecting Mode II traction, $\tau$, with fracture toughness, $G_{I I}$ and displacement $\delta^{*}$, which is the crack sliding displacement at the initial pre-crack tip. For obtaining the nonlinear traction-separation law described by Eq. 2, the relationship $G_{I I}\left(\delta^{*}\right)$ is required. In fact $G_{I I}\left(\delta^{*}\right)$ can be implicitly defined by combining functions $G_{I I}(\alpha)$ and $\delta^{*}(\alpha)$. Functions $G_{I I}(\alpha)$ have already been defined in Fig. 5 for each data reduction scheme, whereas function $\delta^{*}(\alpha)$ was defined studying the recordings of the high resolution camera and using image processing techniques. Thus, the $G_{I I}\left(\delta^{*}\right)$ discrete data sets were plotted for each data reduction scheme and an analytical function was used to fit the results for each case. This procedure is necessary for deriving the differential relationship described in Eq. 2 . 
Table 1. Fracture toughness $G_{\text {IIc }}(\mathrm{N} / \mathrm{mm})$

\begin{tabular}{ccccccc}
\hline & & $\alpha_{1}=35$ & $\alpha_{2}=37$ & $\alpha_{3}=39$ & $\alpha_{4}=41$ & $\alpha_{5}=43$ \\
\hline \multirow{2}{*}{$\mathrm{CCM}$} & Aver. & 0.72 & 1.70 & 1.85 & 1.78 & 1.77 \\
& COV (\%) & 14.1 & 15.1 & 12.7 & 9.7 & 8.7 \\
\hline \multirow{2}{*}{ CBT } & Aver. & 0.65 & 1.28 & 1.55 & 1.69 & 1.72 \\
& COV (\%) & 7.0 & 5.2 & 3.8 & 2.0 & 2.1 \\
\hline \multirow{2}{*}{ CBBM } & Aver. & 0.66 & 1.21 & 1.55 & 1.74 & 1.85 \\
& COV (\%) & 8.6 & 10.8 & 7.0 & 1.7 & 6.4 \\
\hline
\end{tabular}

Figures 6 to 8 present the respective $G_{I I} \delta^{*}$ data sets together with their corresponding fitting functions. The selected fitting functions are those yielding minimum residuals when used with the least square method for the regression of the data sets.

The discrete experimental values of $G_{I I} \delta^{*}$ reduced with CCM exhibit important variations. Thus, an exponential fitting function was utilized for the regression of these data sets, which exhibits a very good approximation. In contrast, $G_{I I} \delta^{*}$ data sets corresponding to the CBT and CBBM schemes are in very good agreement with respect to their repeatability, as shown in figures 7 and 8. CBT results (Fig. 7) exhibit a low increase rate without clearly reaching a steady state fracture energy value. This behaviour is well described with the use of a power law. In the case of CBBM results, steady state fracture toughness was reached near the final experimentally measured values of $G_{I I}$ and $\delta^{*}$. Thus a second order polynomial function which exhibits a very good approximation was utilized for fitting the corresponding results, as presented in Fig. 8.

With the $G_{I I}\left(\delta^{*}\right)$ functions known, the bridging laws $\tau\left(\delta^{*}\right)$ can be hereafter obtained from Eq. 2. Fig. 9 compares the bridging laws (traction-separation laws) as inferred by CCM, CBT and CBBM data reduction schemes. At crack initiation the shear traction attains a peak value followed by a linear or non-linear decay to zero, which depends on the $G_{I I}\left(\delta^{*}\right)$ function obtained for each case. Finally after a specific value of $\delta^{*}$, shear traction is released denoting the absence of the fibre bridging effect under further crack growth. Apart from the function used to describe the tractionseparation laws, there are two characteristic properties that can be inferred. One is the peak value of the shear traction attained at crack initiation, $\tau_{o}$ and the other is the area under the $\tau\left(\delta^{*}\right)$ curve, $I$. These values have been found to be equal to $37.3 \mathrm{MPa}$ and $1.04 \mathrm{~N} / \mathrm{mm}$ for the CCM scheme, 48.4 $\mathrm{MPa}$ and $1.1 \mathrm{~N} / \mathrm{mm}$ for the CBT and $13.9 \mathrm{MPa}$ and $1.14 \mathrm{~N} / \mathrm{mm}$ for the CBBM. A variation is observed over the peak values of shear, whereas the area integrals are in very good agreement.

\section{Conclusions}

A methodology for the characterization of delamination growth under pure Mode II fracture of laminated composite materials was proposed. Fracture energy was measured from ENF tests with the use of three different data reduction schemes. The R-curves and their respective bridging laws

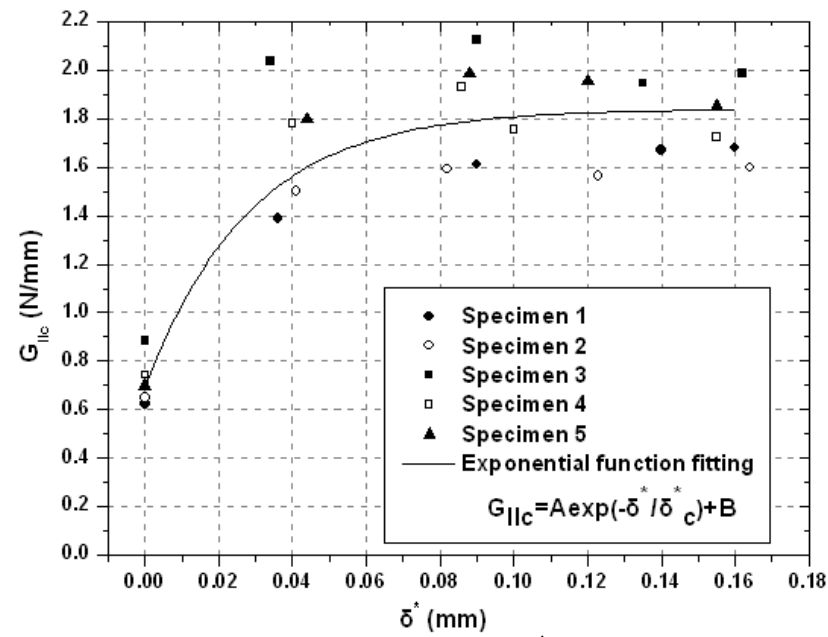

Fig. 6. Experimental $G_{I I}-\delta^{*}$ curve for CCM

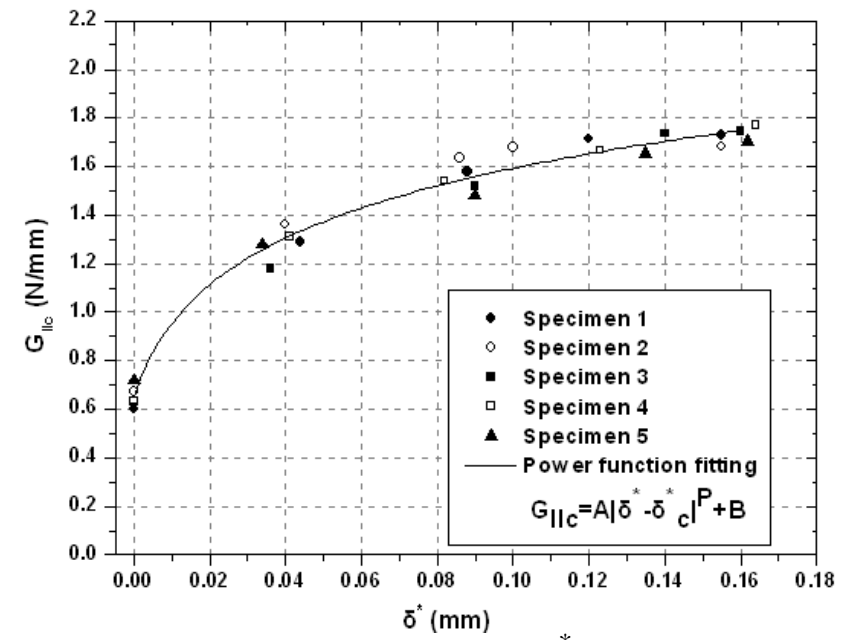

Fig. 7. Experimental $G_{I I} \delta^{*}$ curve for CBT 


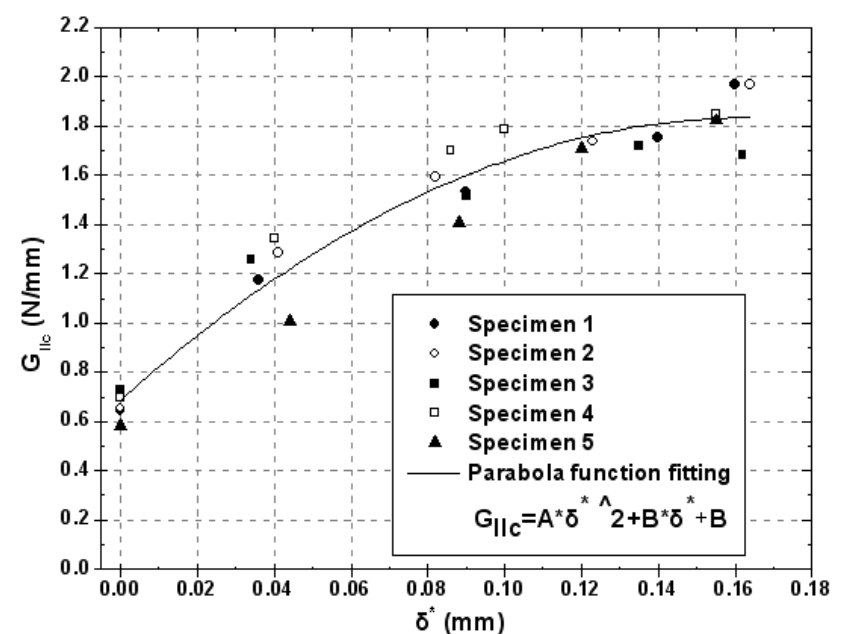

Fig. 8. Experimental $G_{I I} \delta^{*}$ curve for CBBM

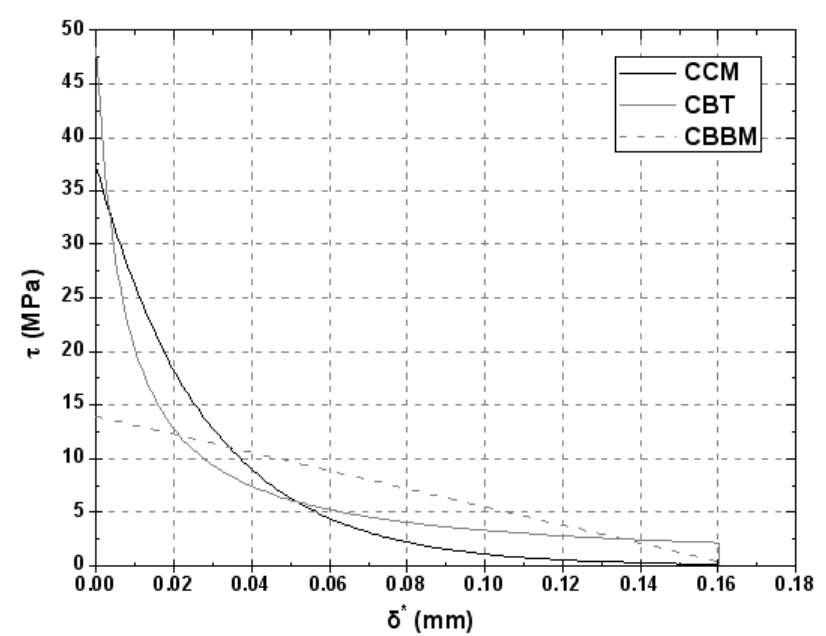

Fig. 9. Experimental bridging laws

were obtained and compared for all three schemes. The obtained bridging laws exhibit the same softening behaviour, regardless the data reduction method used. The energy uptake of the three bridging laws obtained is in great agreement denoting the effectiveness of the proposed procedure in characterizing the FPZ. The laws defined can be used to describe the constitutive relations in interface finite elements for the numerical modelling of delamination growth.

\section{References}

[1] C. Lundsgaard-Larsen, B.F. Sørensen, C.Berggreen and R.C. Østergaard: Engineering Fracture Mechanics Vol. 75 (2008), p. 2514

[2] F.B. Sorensen and T.K. Jacobsen: Composite Science and Technology Vol. 69 (2009), p. 445

[3] J.D. Barrett and R.O. Foschi: Engineering Fracture Mechanics Vol. 9 (1977), p. 371

[4] K.R.B. Blackman, J.A. Brunner and G.J. Williams: Engineering Fracture Mechanics Vol. 73 (2006), p. 2443

[5] H.R. Martin and D.B. Davidson: Rubber and Composites Vol. 28 (1999), p. 401

[6] H. Wang, T. Vu-Khanh and N.V. Le: Journal of Composite Materials Vol. 29 (1995), p. 833

[7] T.K. O'Brien in: Composite Materials: Fatigue and Fracture: 7th Volume, ASTM STP 1330 (1998), p. 3

[8] Z. Suo, G. Bao and B. Fan: Journal of the Mechanics and Physics of Solids Vol. 40 (1992), p. 1

[9] A.L. Carlsson, W.J. Gillespie and B.R. Pipes, R.B.: Journal of Composite Materials Vol. 20 (1986), p. 594

[10] J.A. Russell and N.K. Street, in: Delamination and Debonding of Materials, edited by W.S. Johnson ASTM STP 876, Philadelphia (1985), p. 349

[11] Y. Wang and G.J Williams: Composite Science and Technology Vol. 43 (1992), p. 251

[12]M.F.S.F. de Moura and B.A. de Morais: Engineering Fracture Mechanics Vol. 75 (2008), p. 2584

[13]K. Leffler, S.K. Alfredsson and U. Stigh: International Journal of Solids and Structures Vol. 44 (2007), p. 530 